\title{
Differences in semantic encoding as a function of reading comprehension skill
}

\author{
EDWARD C. MERRILL, RICHARD D. SPERBER, and CHARLEY MCCAULEY \\ Peabody College, Vanderbilt University, Nashville, Tennessee 37203
}

\begin{abstract}
Using a modified Stroop procedure, we examined the extent to which the semantic encoding of a word is governed by the context within which that word appears. Good and poor comprehenders named the color of target words following their reading of either sentences or single words representing the object nouns of the sentences. Target words represented contextually emphasized (appropriate) attributes of the object nouns, nonemphasized (inappropriate) attributes of these nouns, or object attributes not related to these nouns (neutral). For single-word contexts, all subjects exhibited equal semantic interference to appropriate and inappropriate targets, relative to neutral targets. For sentence contexts, however, good comprehenders exhibited semantic interference only to appropriate targets, whereas poor comprehenders again exhibited equal interference to appropriate and inappropriate targets. These findings suggest that differences in comprehension skill may be attributable, at least in part, to fundamental differences in the way in which sentences are semantically encoded.
\end{abstract}

In the experiment reported below, we explored the possibility that individual differences in general comprehension skill are associated with fundamental differences in the way that words are semantically encoded. We were specifically interested in determining whether skilled and less skilled comprehenders differ in the extent to which the semantic encoding of a word is governed by the context within which that word appears.

The data currently available indicate that the semantic encoding operations used by skilled and less skilled comprehenders are comparable when these readers process familiar words in isolation (e.g., Golinkoff \& Rosinski, 1976; Merrill, Sperber, \& McCauley, 1980). However, when a word is part of a meaningful sentence, contextual constraints are present that surely influence the encoded meaning of that word. The ability to use context in this fashion may involve semantic encoding operations that are more complex or elaborate than those required for the processing of words in isolation. For this reason, group differences in semantic encoding processes that are absent when words are processed in isolation may emerge when these same words are processed as part of a sentence.

This research was supported by Grants HD-00973, HD-07045, and HD-04510 from the National Institute of Child Health and Human Development. The authors wish to thank Thomas Carr for his valuable comments on an earlier draft of this manuscript. We are also grateful to Robert Cantrell and the teachers of Fairview Elementary School for their active cooperation and support. A report of this research was presented at the Southeastern Conference on Human Development, Alexandria, Virginia, April 1980. Requests for reprints should be sent to Richard Sperber, Peabody Box 512, Vanderbilt University, Nashville, Tennessee 37203.
Our view of context-specific encoding is largely based on the notion of "semantic flexibility" as proposed by Barclay and his associates (Barclay, Bransford, Franks, McCarrell, \& Nitsch, 1974). Barclay et al., using a cued recall procedure, obtained data indicating that the relative salience of a word's semantic features, and, hence, the precise nature of the encoded representation of that word, is determined by the context supplied by the sentence within which the word appears. For example, although a piano is both a heavy object and a musical object, when the word "piano" was presented in the sentence "The man lifted the piano," cues that referred to the heaviness of the piano were more effective in eliciting recall of the final noun than were cues that referred to the musical nature of a piano. The opposite pattern of data was obtained when "piano" was presented in the sentence "The man tuned the piano." Based on the logic of the cued recall procedure (cf. Thomson \& Tulving, 1970; Tulving \& Thomson, 1973), Barclay et al. concluded that it is the contextually determined salience of a word's semantic features, rather than the static, objective salience of those features, that is emphasized in the encoded representations of words presented in sentences. Subsequent research has indicated that this context-specific encoding may also characterize the processing of subject nouns and verbs (e.g., R. Anderson \& Ortony, 1975; R. Anderson, Pichert, Goetz, Shallert, Stevens, \& Trollip, 1976).

In the present study, we tested the hypothesis that the encoding processes used by skilled and less skilled comprehenders are differentially sensitive to the biasing effects of sentence contexts. More specifically, we predicted that the encoding operations used by skilled comprehenders result in semantic representations that 
emphasize the context-specific salience of word attributes, whereas the encoding operations of less skilled comprehenders result in representations that more closely reflect the objective salience of word attributes.

To avoid the potentially confounding effects of group differences in memory processes and allow the separation of encoding from retrieval phases of comprehension, we tested the above predictions using a modified Stroop procedure similar to that used by Conrad (1974). Good and poor readers were shown target words printed in colored ink that were preceded by either sentence or word contexts. Subjects were required to read the context stimulus and then to name the color of the target as rapidly as possible. Target words were object attributes that were selected so as to vary in their degree of appropriateness to the sentence contexts with which they were paired. These pairings formed appropriate relationships (e.g., "The girl touched the cat-fur" and "The girl fought the cat-claw"), inappropriate relationships (e.g., "The girl touched the cat-claw" and "The girl fought the cat-fur"), and neutral relationships (e.g., "The man washed the car-fur" and "The man fixed the car-claw"). These same relationships were maintained for the pairing of targets with word contexts by simply presenting the isolated object noun of each sentence with the same target that appeared with that sentence in the sentence conditions (e.g., "cat-fur" and "cat-claw," "cat-claw" and "cat-fur," and "car-fur" and "car-claw" for the appropriate, inappropriate, and neutral conditions, respectively).

Under similar task conditions, color-naming latencies to target words have been shown to vary with the degree of semantic relatedness between the target and the preceding context stimulus; that is, longer latencies are obtained when the target is semantically related to the preceding stimulus than when the two stimuli are unrelated (e.g., Warren, 1972). In the present experiment, the magnitude of interference obtained in the appropriate and inappropriate conditions, relative to that obtained in the neutral condition, was assumed to reflect the degree to which the semantic attributes represented by the target words had been included in the encoded representation of the preceding context stimuli. Thus, while color-naming latencies to appropriate and inappropriate targets were expected to be longer than those to neutral targets for all subjects, group differences were expected as a function of whether subjects had read single-word or sentence contexts. No group differences in interference effects were expected following the reading of single words, with equal interference being obtained for appropriate and inappropriate targets. However, to the extent that good comprehenders more effectively utilize contextual constraints to influence the semantic encoding of words during sentence processing, different patterns of interference would be expected to obtain for these groups following the reading of sentences. For good comprehenders, whose encodings were expected to emphasize the contextually determined salience of a word's semantic attributes, we expected to observe significantly greater interference in the appropriate than in the inappropriate sentence condition. In comparison, we predicted that for poor comprehenders, the appropriateinappropriate difference would be relatively slight, if present at all, since their encoded representations were expected to be based primarily on the objective salience of word attributes.

\section{METHOD}

\section{Subjects}

The subjects were 14 good and 14 poor comprehenders selected from fifth-grade classes at a predominately white, middle-class, rural public school. Group assignment was made on the basis of scores achieved on the comprehension subtest of the Stanford Achievement Test. Good comprehenders were defined as students who had achieved a grade-equivalent score of 5.0 or higher, and poor comprehenders were defined as students who had achieved a score of 4.5 or lower on this subtest. Mean grade-equivalent scores were $7.5(\mathrm{SD}=1.3)$ for the good comprehenders and $3.5(\mathrm{SD}=.9)$ for the poor comprehenders. The corresponding mean percentile ranks were $88 \%$ (range $=68 \%-96 \%$ ) and $18 \%$ (range $=12 \% 30 \%$ ). In an effort to better classify the particular readers used in this study, scores were also obtained for the vocabulary, spelling, word study, and language subtests. Mean gradeequivalent scores for good and poor comprehenders, respectively, were 6.5 and 3.6 in vocabulary, 7.2 and 3.5 in spelling, 6.9 and 4.2 in word study, and 7.1 and 3.5 in language.

\section{Design and Materials}

The experimental conditions, along with examples of the stimuli used in each condition, are presented in Table 1. The variables were groups (good and poor comprehenders), context type (sentences and words), and relatedness (appropriate, inappropriate, and neutral). Levels of the relatedness factor were designated on the basis of the semantic relationship between the

Table 1

Examples of Context-Target Combinations Presented in the Sentence Context and Word Context Conditions

\begin{tabular}{|c|c|c|}
\hline \multirow[b]{2}{*}{ Condition } & \multicolumn{2}{|l|}{ Stimulus Example } \\
\hline & Context & Target \\
\hline \multicolumn{3}{|c|}{ Sentence Context } \\
\hline Appropriate & $\begin{array}{l}\text { The girl touched the cat. } \\
\text { The girl fought the cat. }\end{array}$ & $\begin{array}{l}\text { fur } \\
\text { claw }\end{array}$ \\
\hline Inappropriate & $\begin{array}{l}\text { The girl touched the cat. } \\
\text { The girl fought the cat. }\end{array}$ & $\begin{array}{l}\text { claw } \\
\text { fur }\end{array}$ \\
\hline Neutral & $\begin{array}{l}\text { The man washed the car. } \\
\text { The man fixed the car. }\end{array}$ & $\begin{array}{l}\text { fur } \\
\text { claw }\end{array}$ \\
\hline Appropriate & $\begin{array}{l}\text { Word Context } \\
\text { cat } \\
\text { cat }\end{array}$ & $\begin{array}{l}\text { fur } \\
\text { claw }\end{array}$ \\
\hline Inappropriate & $\begin{array}{l}\text { cat } \\
\text { cat }\end{array}$ & $\begin{array}{l}\text { claw } \\
\text { fur }\end{array}$ \\
\hline Neutral & $\begin{array}{l}\text { car } \\
\text { car }\end{array}$ & $\begin{array}{l}\text { fur } \\
\text { claw }\end{array}$ \\
\hline
\end{tabular}


sentence contexts and their targets. An appropriate relationship was defined as one in which the target word represented an implicitly emphasized semantic feature of the object noun of the sentence. An inappropriate relationship was defined as one in which the target word represented a nonemphasized semantic feature of the object noun of the sentence. A neutral relationship was defined as one in which the target word was unrelated to the object noun.

Stimuli for the three sentence conditions were formed in the following manner. Six pairs of subject-verb-object (S-V-O) sentences were generated such that the members of each pair differed from each other only at the verb position. Each sentence was then assigned a target word that met the above criterion for an appropriate relationship. The resulting 12 contexttarget combinations formed the stimuli for the appropriatesentence condition. The stimuli for the inappropriate-sentence condition were constructed by re-pairing the context and target stimuli within each of the six sentence pairs. Twelve additional $\mathrm{S}-\mathrm{V}-\mathrm{O}$ sentences were generated and randomly paired with these same targets to form the context-target combinations for the neutral-sentence condition. The entire set of sentence context-target pairings are presented in Table 2 .

The context-target combinations for the word conditions were formed by simply pairing the isolated object noun of each sentence with the same target that appeared with that sentence in the sentence conditions. In this manner, 36 contexttarget combinations were formed for the single-word conditions, 12 in each of the three relatedness conditions. As can be seen in Table 1, the appropriate and inappropriate conditions generated in this manner were identical in the word conditions. This was done to facilitate data analysis, in that context type and relatedness were fully crossed.

As mentioned above, the subjective salience of the object noun's semantic features in the sentence conditions was manipulated by simply changing the verb of the sentence, with all other components of the sentence remaining unchanged. Thus, it was conceivable that any response time differences as a function of relatedness could be attributed to the direct influence of the verbs on the color-naming of appropriate vs. inappropriate targets, without requiring any assumptions about the differential encoding of object nouns. In order to assess the independent effect of the verbs on the color naming of appropriate vs. inappropriate targets, 24 additional context-target combinations (12 appropriate and 12 inappropriate) were included in which the verb of each sentence, like the object noun, was presented in isolation as a context stimulus for both the appropriate and inappropriate targets.

Stimuli were typed in the center of transparent slides for presentation. Sentences began with a capital letter and ended with a period. Single words were typed in lowercase. All of the context stimuli were typed in black ink, and the target words were typed in red, blue, green, or brown ink. The assignment of colors to particular targets was made such that each target word appeared twice in each color, and across targets, each color appeared equally often in each condition. In this manner, a total of 96 context-target combinations were constructed, 12 in each of the eight experimental conditions, with each target word appearing once in each condition. An additional set of 24 context-target combinations was constructed in a similar manner for use during practice, with the exception that all combinations constructed for practice involved neu tral relationships.

The 96 context-target combinations were divided into two blocks such that each block contained an equal number of stimuli from each condition and each target word appeared four times in each block. The order of stimuli within each block was randomized. Each subject was tested individually in two sessions, with the interval between sessions being approximately 2 weeks. Subjects received one block of trials per session. The presentation order of trial blocks was counterbalanced across subjects within groups.

\section{Apparatus}

The apparatus consisted of two Kodak Carousel (Model C) slide projectors equipped with tachistoscopic lenses for presenting stimuli, a voice-operated relay wired to a Hunter Model $120-\mathrm{C}$ Klockounter for recording response times to targets, and supportive electromechanical equipment. The Klockounter was interfaced with the voice-operated relay and the tachistoscopic lens of one of the projectors such that the onset of the target initiated the timing cycle and the subject's response to the target stopped the cycle.

\section{Procedure}

At the beginning of each session, the subject was first shown the four target colors and was asked to name each one. This was done to ensure that subjects would assign a distinct label to each color. Subjects were allowed to use any label for a given color that they spontaneously chose, but they were required to use the same four labels throughout the experiment. The subject was then given practice in naming the colors of typed words. Twenty-four single words were presented, and the subject was instructed to name the color of each as rapidly as possible without error. This was followed by the presentation of 24 practice trials that were identical in format to the experimental trials.

For each practice and experimental trial, the context stimulus (sentence or word) first appeared on the screen and the subject read the sentence or word aloud. Immediately

Table 2

Sentence Context/Target Combinations Used in the Appropriate, Inappropriate, and Neutral Sentence Conditions

\begin{tabular}{lll}
\hline Target & \multicolumn{1}{c}{ Appropriate } & Sentence Context \\
\hline $\begin{array}{l}\text { fur } \\
\text { claw }\end{array}$ & The girl touched the cat. & The girl fought the cat. \\
fire & The man saw the smoke. & The girl fought the cat. \\
cigar & The man blew the smoke. & The man blew the smoke. \\
music & The man played the piano. & The man saw the smoke. \\
heavy & The man moved the piano. & The man moved the piano. \\
smell & The boy held his nose. & The man played the piano. \\
sniffle & The boy blew his nose. & The boy blew his nose. \\
witch & The woman pushed her broom. & The boy held his nose. \\
Tarm & The woman flew her broom. & The woman flew her broom. \\
smoke & The boy sat near the fire. & The woman pushed her broom. \\
\hline
\end{tabular}


following the subject's reading response, the context stimulus was removed, and $1 \mathrm{sec}$ later, the target word appeared, initiating the timing cycle. The subject named the color of the target word as rapidly as possible, and this naming response stopped the timing cycle and ended the trial. Response times were recorded to the nearest $1 \mathrm{msec}$.

To insure that all subjects processed the semantic content of the context stimuli, a recognition memory test was given following target naming on $25 \%$ of the experimental trials. On these 24 randomly selected trials, the subject was presented black-andwhite line drawings of scenes (for the sentences) or objects (for the words) and was asked to verify whether or not the picture accurately depicted the context stimulus for the trial. Half of these drawings accurately depicted the context stimulus and half did not. Inaccurate depictions of sentence contexts were constructed such that the same objects referred to in the sentences were represented in the scenes, but the relationships among them were incorrect.

\section{RESULTS}

\section{Picture Recognition}

Performance on the picture recognition task was nearly perfect for all subjects. Twenty-one of 28 subjects committed no errors, and the remaining 7 ( 4 good and 3 poor comprehenders) committed one error each. While these data would clearly be of little value in assessing group differences in memory for the context stimuli, the data do serve the purpose intended here of indicating that both groups semantically processed the context stimuli and retained the context in memory at least until the completion of the trial.

\section{Color-Naming Latencies}

Since the verb-context conditions were included for control purposes and did not cross factorially with the variables of primary interest, response times in these conditions were analyzed separately, as described below. Mean response times (excluding errors) in the remaining conditions were analyzed in separate groups (good and poor comprehenders) by context type (sentences and words) by relatedness (appropriate, inappropriate, and neutral) mixed analyses of variance, alternately treating subjects and items as the random variable. Since preliminary analysis had shown that response times did not differ as a function of sessions or presentation order of blocks, these factors were not included in the primary analyses. Errors were relatively rare $(2.5 \%$ for good comprehenders and $5.0 \%$ for poor comprehenders), and no reliable trend in error rates as a function of groups, or conditions within groups, was observed (see Figure 1).

The primary analyses revealed significant main effects of context type $\left[F_{1}(1,26)=11.78, p<.01 ; F_{2}(1,22)=\right.$ $11.86, \mathrm{p}<.01]$ and relatedness $\left[\mathrm{F}_{1}(2,52)=10.01\right.$, $\left.p<.01 ; \quad F_{2}(2,44)=7.59, p<.01\right]$. The effect of context type resulted from longer response times to targets preceded by sentence contexts relative to those preceded by word contexts ( 944 vs. $893 \mathrm{msec}$, respectively). This difference was apparent for neutral targets as well as related targets, indicating that some general, non- semantic component of sentence and word processing must account for this effect. We are not sure what that component might be. One possibility is that for the more complex sentence materials, relatively greater effort is required for comprehending and for retaining in memory, at least briefly, the outcome of the comprehension process. Relatively less processing energy or capacity would thus be left for target processing, resulting in a relative increase in response times. This argument would suggest that the context effect should dissipate with longer context-target intervals. The main effect of relatedness is best understood in relationship to the significant three-way interaction of Groups by Context Type by Relatedness $\left[\mathrm{F}_{1}(2,52)=3.59, \mathrm{p}<.05\right.$; $\left.\mathrm{F}_{2}(2,44)=3.87, \mathrm{p}<.05\right]$. To facilitate the interpretation of this interaction, we examined the effects of groups and relatedness separately for word and sentence contexts.

Word contexts. Mean response times in the wordcontext conditions are presented in Figure 1. A groups by relatedness analysis of variance revealed a significant main effect of relatedness $\left[F_{1}(2,52)=3.38, p<.05\right.$;

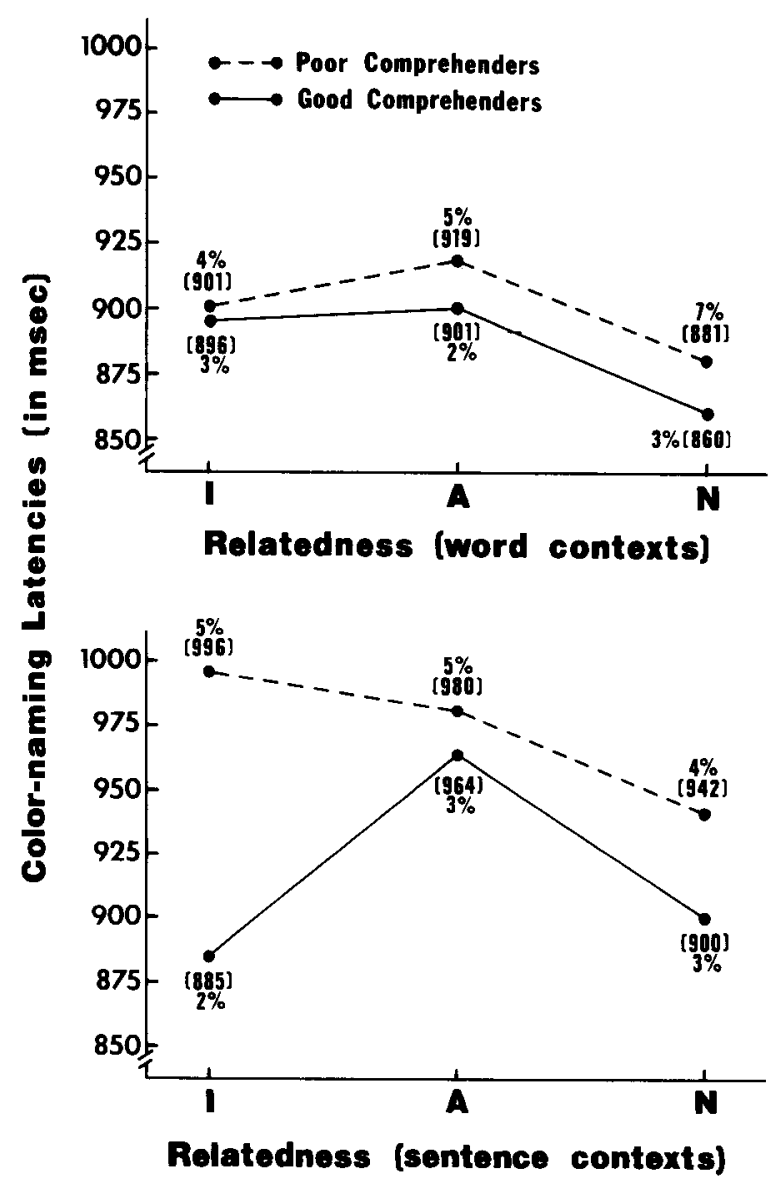

Figure 1. Mean color-naming latencies and error rates for targets preceded by word contexts (top) and sentence contexts (bottom) as a function of groups and relatedness $(\mathrm{I}=$ inappropriate; $A$ = appropriate; $N=$ neutral). 
$\left.\mathrm{F}_{2}(2,44)=3.54, \mathrm{p}<.05\right]$, and orthogonal comparisons were conducted to determine the source of the effect. Response times in the appropriate condition did not differ from those in the inappropriate condition $\left[F_{1}(1,26)=.59 ; F_{2}(1,22)=.37\right]$; this was as expected, since the appropriate-inappropriate manipulation served as a dummy variable for single-word contexts. However, response times for appropriate and inappropriate targets combined were significantly longer $(904 \mathrm{msec})$ than those for neutral targets $(870 \mathrm{msec})\left[\mathrm{F}_{1}(1,26)=6.16\right.$; $\left.p<.05 ; F_{2}(1,22)=6.70, p<.05\right]$. Yet, neither the main effect of groups nor the groups by relatedness interaction even approached significance $\left[F_{1}(1,26)=\right.$ $.18 ; \mathrm{F}_{2}(1,22)=.54 ;$ and $\mathrm{F}_{1}(2,52)=.19, \mathrm{~F}_{2}(2,44)=.08$, respectively]. Thus, these good and poor comprehenders exhibited significant and equal interference to targets preceded by related single-word contexts, a finding that is consistent with those of Golinkoff and Rosinski (1976) and Merrill et al. (1980) in suggesting comparable semantic encoding of single words by good and poor readers.

Sentence contexts. As can be seen in Figure 1, the pattern of response times obtained with sentence contexts was quite different for the good and poor comprehenders. The main effect of groups was significant only with items treated as the random variable $\left[F_{1}(1,26)=1.79 ; F_{2}(1,22)=7.20, p<.05\right]$. However, the main effect of relatedness $\left[F_{1}(2,52)=6.96, p<.01\right.$; $\left.F_{2}(2,44)=5.18, p<.01\right]$ and the interaction of Groups by Relatedness $\left[F_{1}(2,52)=6.16, p<.01 ; F_{2}(2,44)=\right.$ $5.28, \mathrm{p}<.01]$ were reliable for both items and subjects. Subsequent analyses revealed significant relatedness effects for both good comprehenders $\left[F_{1}(2,26)=9.29\right.$, $\left.\mathrm{p}<.01 ; \mathrm{F}_{2}(2,22)=6.02, \mathrm{p}<.01\right]$ and poor comprehenders $\left[F_{1}(2,26)=3.92, p<.05 ; \quad F_{2}(2,22)=3.89\right.$, $\mathrm{p}<.05]$, although the locus of these effects clearly differed for the two groups.

For good comprehenders, color-naming latencies to appropriate targets were substantially longer than those to both inappropriate and neutral targets (both ps $<.01$ using Newman-Keuls), clearly indicating that, consistent with our initial predictions, these subjects semantically encoded the object nouns of sentences in accordance with the contextually determined salience of the nouns' semantic features. Of additional interest was the finding that response times in the neutral and inappropriate conditions did not differ for the good comprehenders, suggesting that the biasing effect of sentence context was so robust for these subjects that little, if any, information about the nonemphasized features of the object nouns was included in the encoded representations of these words.

In contrast, the pattern of data obtained for poor comprehenders with sentence contexts was essentially identical to that obtained with single-word contexts. Once again, response times in the appropriate and inappropriate conditions did not differ from each other $\left[F_{1}(1,26)=.64 ; F_{2}(1,22)=.91\right]$, but the mean response time for the two related conditions combined was signif- icantly slower than that obtained in the neutral condition $\left[F_{1}(1,26)=7.20, p<.01 ; F_{2}(1,22)=6.86\right.$, $\mathrm{p}<.05]$. Thus, the indication is that, unlike the good comprehenders, these subjects semantically encoded the object nouns of sentences in the same way they encoded these words in isolation: in accordance with the objective salience of the words' semantic features.

\section{Verb Control Conditions}

Mean color-naming latencies in the verb conditions were analyzed in a groups by relatedness (appropriate vs. inappropriate) mixed analysis of variance. As expected, none of the effects in the analysis even approached significance (all Fs $<1.0$ ). Of major importance, the isolated verbs did not differentially influence response times in the appropriate and inappropriate conditions (924 and $933 \mathrm{msec}$, respectively). These results indicate that the different patterns of data observed for good and poor comprehenders in the sentence conditions cannot be attributed to group differences in the direct influence of the verbs on the color-naming latencies of targets.

In an effort to partially control for the problem of potential group differences in general intellectual functioning, all analyses were redone using a subset of the original groups (10 good comprehenders and 10 poor comprehenders) after matching for mathematical ability (grade-equivalent scores of 5.1 and 4.9 on Total Math for the good and poor comprehenders, respectively). The pattern of response times and the results of the analyses were essentially identical to those reported above. All significant effects reported for the word contexts were maintained, and for the good comprehenders, the results likewise were identical. For the poor comprehenders, one of the reported results (the effect of relatedness for sentence contexts) was only marginally significant $(p<.07)$. However, with orthogonal comparisons, the effects of primary interest were significant here, as well.

In summary, then, the primary results were quite consistent with the basic hypotheses. Object nouns interfered with the color-naming of targets that represented semantic features of the objects, relative to neutral targets, to the same degree for good and poor comprehenders. The isolated verbs did not differentially interfere with the color naming of appropriate and inappropriate targets for either group. However, when these same nouns and verbs were presented in sentences, good comprehenders exhibited semantic interference that was context specific (i.e., only to appropriate targets), whereas poor comprehenders exhibited interference to the inappropriate targets that was equal in magnitude to that for the appropriate targets.

\section{DISCUSSION}

The task before us, then, is to determine the nature of the semantic representations encoded during sentence comprehension that would yield the obtained pattern of data. Clearly, the encoded representations con- 
structed by good and poor comprehenders were sufficient to enable both groups to accurately recognize pictorial representations of the experimental sentences. However, the patterns of semantic interference obtained in the color-naming task imply that the semantic codes used to verify pictures were, in fact, different for the two groups.

The pattern of semantic interference exhibited by the good comprehenders (i.e., more interference to appropriate than to inappropriate targets) may suggest that these readers rely on sentence representations that are constructed on the basis of holistic or configural properties of the sentence (cf. Barclay et al., 1974; Carr \& Bacharach, 1978; Till, 1977). That is, the meaning of the sentence as a whole determines the manner in which individual words and word concepts are encoded, with the result that contextual information is incorporated directly into the functional units of the overall representation. Thus, information about specific word concepts that is irrelevant and inappropriate to the meaning of the sentence as a whole would not be included in the representations of the sentence. Indeed, these characteristics of the sentence code suggest that the basic unit of the sentence representation would be at the level of the phrase or sentence, rather than at the level of individual words or word concepts.

In contrast, the pattern of semantic interference exhibited by the poor comprehenders (i.e., equal interference to appropriate and inappropriate targets) indicates that the basic units of the semantic representations encoded for sentences by these readers are, in fact, the individual words and word concepts. This contention is further supported by the similarity in the patterns of semantic interference exhibited by this group to targets preceded by words in isolation and those preceded by these same words in sentences. Presumably, then, in order to comprehend and semantically represent the sentence, the individual concepts must be bound together into a single representation, possibly in a manner similar to that suggested by the associative network model of J. Anderson and Bower (1973). Within this framework, the encoded information necessary for correct responding on our recognition memory test would be represented by associative links that specify the relations between individual words. The encoded representations of the words themselves, however, would remain invariant across different contexts. Comprehension and memory would thus depend upon the construction and maintenance of a complete network of associations, since each component of the overall representation supplies the reader with unique information.

The reliance of poor comprehenders on an associative rather than integrated representation of the sentence would have several disadvantages. For example, an associative network representation, in which the basic units are the individual word concepts, might tend to bind the poor comprehender to exact wordings. Such a state of affairs would seriously limit the ability of these readers to paraphrase or retain the gist of a sentence as individual words are forgotten-a prediction that receives some support from the findings of Perfetti and Goldman (1976; see also Perfetti \& Lesgold, 1977). In addition, it seems reasonable to suggest that, since the semantic representation of the sentence encoded by poor comprehenders would be little more than a duplication of the original sentence, these readers would find it necessary to reinterpret the meaning of the sentence during retrieval, just as it was necessary to interpret the meaning during input. In contrast, the semantic representation encoded by good comprehenders would actually incorporate the specified interpretation, and thus, reinterpretation during retrieval would not be required. This difference between groups would likely be manifested in the ease and efficiency with which good and poor comprehenders are able to retrieve and/or manipulate linguistic information currently held in working memory (cf. Kail, Chi, Ingram, \& Danner, 1977). In a similar vein, if contextually invariant word concepts serve as the basic units of the sentence representation for poor comprehenders, then one might expect these readers to have a tendency to confuse sentences that contain the same set of words. That is, these readers may tend to passively associate two or more sentences together by virtue of the overlap in word concepts that exist within the semantic represen. tations of the sentences. Good comprehenders, whose encodings of word concepts vary with context, would be less likely to exhibit such confusions, except in those circumstances in which the contexts supplied by the sentences and, hence, the interpretations of the individual words were in fact similar. Indeed, group differences in the propensity to make this type of semantic confusion may help to account for the finding that poor readers exhibited a higher degree of "semantic integration" than did good readers when asked to remember sentences verbatim (cf. Waller, 1976).

Finally, the differential reliance on one or the other of these semantic codes during individual sentence processing should have consequences for comprehension at the more global "macrostructural" level (e.g., Kintsch \& van Dijk, 1978; Kintsch \& Vipond, 1979). At this level of processing, the unit of comprehension is assumed to be the macroproposition, which is constructed from the semantic codes of individual sentences (micropropositions). The efficient reader examines the available micropropositions and designates each as either relevant or irrelevant. Relevant micropropositions are then used to form new macropropositions or to modify existing ones; irrelevant micropropositions are discarded. Within the current framework, determining the relevance of a given microproposition would necessarily be more difficult for poor comprehenders by virtue of their inclusion of irrelevant and inappropriate semantic information within the micropropositions. Thus, the efficiency 
with which poor comprehenders are able to utilize macrolevel processes may be limited, at least in part, by the inefficient execution of microlevel processes.

On the surface, the data reported for our good comprehenders may appear inconsistent with that reported by Conrad (1974), who used a similar procedure. Conrad found that a biasing context did not influence the manner in which college students processed words with two distinct meanings. However, it is important to note that Conrad's interest was in the effects of context on lexical access. Hence, the activation of semantic pathways was assessed immediately following sentence presentation. Since our interest was in context effects that occur after lexical access during comprehension, we selected a delay interval of $1 \mathrm{sec}$ between context offset and target onset. It is during this period of active comprehension, rather than at the time of lexical access, that context-specific selection and encoding operations most likely occur, according to the recent data and theory presented by Swinney and his associates (Swinney, 1979; Swinney \& Hakes, 1976). They have shown that very brief intervals between the presentation of a word in context and the measurement of patterns of semantic activation are critical to the observation of context-specific encoding effects. With respect to our good and poor comprehenders, then, we envision a framework for sentence comprehension in which both groups would exhibit relatively automatic activation of all semantic pathways during lexical access. While poor comprehenders do not appear to continue processing beyond this point, good comprehenders continue processing up to and perhaps beyond the point at which context-specific encoding operations override automatic activation.

\section{REFERENCES}

Anderson, J. R., \& Bowen, G. H. Human associative memory. Washington, D.C: Winston, 1973.

Anderson, R. C., \& Ortony, A. On putting apples into bottlesA problem of polysemy. Cognitive Psychology, 1975, 7, 167-180.

Anderson, R. C., Pichert, J. W., Goetz, E. T., Shallert, D. L., Stevens, K. V., \& Trollip, S. R. Instantiation of general terms. Journal of Verbal Learning and Verbal Behavior, 1976, 15, 667-679.

Barclay, J., Brangford, J., Franks, J., McCarrell, N., \&
Nitsch, K. Comprehension and semantic flexibility. Journal of Verbal Learning and Verbal Behavior, 1974, 13, 471-481.

Carr, T. H., \& Bacharach, V. R. Developmental differences in remembering sentences after integrative or selective semantic processing. Journal of Experimental Child Psychology, 1978, 26, 443-457.

Conrad, C. Context effects in sentence comprehension: A study of the subjective lexicon. Memory \& Cognition, 1974, 2, 130138.

Golinkoff, R. M., \& Rosinskı, R. R. Decoding, semantic processing, and reading comprehension skill. Child Development, 1976, 47, 252-258.

Kail, R. V., Chi, M. T. H., Ingram, A. L., \& Danner, F. W. Constructive aspects of children's reading comprehension. Child Development, 1977, 48, 684-688.

Kint8ch, W., \& van DiJK, T. A. Toward a model of text comprehension and production. Psychological Review, 1978, 85, 363-394.

KINTSCH, W., \& VIPOND, D. Reading comprehension and readability in educational practice and psychological theory. In L.-G. Nilsson (Ed.), Perspectives on memory research. Hillsdale, N.J: Erlbaum, 1979.

Merrill, E. C., Sperber, R. D., \& McCauley, C. The effects of context on word identification in good and poor readers. Journal of Psychology, 1980, 106, 179-192.

Perfett, C. A., \& Goldman, S. R. Discourse processing and reading comprehension skill. Journal of Verbal Learning and Verbal Behavior, 1976, 14, 33-42.

Perfetti, C. A., \& Lesgold, A. M. Discourse comprehension and sources of individual differences. In M. A. Just \& P. A. Carpenter (Eds.), Cognitive processes in comprehension. Hillsdale, N.J: Erlbaum, 1977.

Swinney, D. A. Lexical access during sentence comprehension: (Re)Consideration of context effects. Journal of Verbal Learning and Verbal Behavior, 1979, 18, 645-659.

Swinney, D. A., \& HAkEs, D. T. Effects of prior context upon lexical access during sentence comprehension. Journal of Verbal Learning and Verbal Behavior, 1976, 15, 681-689.

Thomson, D. M., \& Tulving, E. Associative encoding and retrieval: Weak and strong cues. Journal of Experimental Psychology, 1970, 86, 255-262.

TiLl, R. E. Sentence memory prompted with inferential recall cues. Journal of Experimental Psychology: Human Learning and Memory, 1977, 3, 129-141.

Tulving, E., \& Thomson, D. M. Encoding specificity and retrieval processes in episodic memory. Psychological Review, 1973, 80, 352-373.

WAller, T. G. Children's recognition memory for sentences: A comparison of good and poor readers. Child Development, $1976,47,90-95$.

WARREN, R. E. Stimulus encoding and memory. Journal of Experimental Psychology, 1972, 94, 90-100.

(Received for publication October 9, 1980; revision accepted June 8, 1981.) 